\title{
Effective Bounds for the Maximal Order of an Element in the Symmetric Group
}

\section{By Jean-Pierre Massias, Jean-Louis Nicolas, and Guy Robin*}

\begin{abstract}
Let $\sigma_{n}$ be the symmetric group of $n$ elements and

$$
g(n)=\max _{\sigma \in \sigma_{n}}(\text { order of } \sigma) \text {. }
$$

We give here some effective bounds for $g(n)$ and $P(g(n))$ (greatest prime divisor of $g(n)$ ). Theoretical proofs are in "Evaluation asymptotique de l'ordre maximum d'un élément du groupe symétrique" (Acta Arith., v. 50, 1988, pp. 221-242).

The tools used here are techniques of superior highly composite numbers of Ramanujan and bounds of Rosser and Schoenfeld on the Chebyshev function $\theta(x)$.
\end{abstract}

1. Introduction. Let $g(n)$ denote the greatest order of a permutation in the symmetric group $\sigma_{n}$. It seems that $g(n)$ was first considered by E. Landau (cf. [1] and [2]), who proved

$$
\log g(n) \sim \sqrt{n \log n} .
$$

W. Miller recently wrote a survey paper which summarizes what is known about $g(n)$ (cf. [7]), and very recently, we gave in [5] an improvement of (1.1), namely

$$
\log g(n)=\sqrt{\mathrm{li}^{-1}(n)}+O(\sqrt{n} \exp (-a \sqrt{\log n})) \quad(a>0),
$$

where $\operatorname{li}(x)$ is the logarithmic integral.

An easy consequence of (1.2) is (cf. [5, pp. 225])

$$
\begin{aligned}
& \log g(n) \\
& =\sqrt{n \log n}\left(1+\frac{\log \log n-1}{2 \log n}-\frac{(\log \log n)^{2}-6 \log \log n+9+o(1)}{8 \log ^{2} n}\right) .
\end{aligned}
$$

In [3], it is proved that

$$
\max _{n \geq 1}(\log g(n)) / \sqrt{n \log n}=1.05313 \ldots
$$

holds, with the maximum assumed for $n=1,319,166$. In [4], it is proved that

$$
\log g(n) \leq \sqrt{n \log n}\left(1+\frac{\log \log n}{2 \log n}\right)
$$

Received June 27, 1988; revised November 29, 1988.

1980 Mathematics Subject Classification (1985 Revision). Primary 11N37, 11Y60, $20 \mathrm{~B} 30$.

Key words and phrases. Symmetric group, arithmetic function, highly composite numbers.

* Research supported by "Centre National de la Recherche Scientifique", P.I.C.S. "Utilisation des ordinateurs en théorie des Nombres", G.R.E.C.O. "Calcul Formel", P.R.C.: "Mathématiques, Informatique". 
for all $n \geq 2$ and that

$$
\log g(n) \geq \sqrt{n \log n}
$$

is valid for $n \geq 906$.

The aim of this paper is to prove the following improvements of $(1.4),(1.5)$ and (1.6).

THEOREM 1. The assertion

$$
n \geq n(b) \Rightarrow \log g(n) \geq \sqrt{n \log n}\left(1+\frac{\log \log n-b}{2 \log n}\right)
$$

is valid for the following pairs $(b, n(b))$ :

\begin{tabular}{|c|c|c|c|c|c|c|c|}
\hline$b$ & 2 & 1.9 & 1.8 & 1.7 & 1.6 & 1.5 & 1.4 \\
\cline { 1 - 7 }$n(b)$ & 810 & 1,201 & 1,530 & 3,896 & 7,228 & 17,595 & 37,402 \\
\hline$b$ & 1.3 & 1.2 & 1.19 & 1.18 & 1.17 & 1.16 & \\
\cline { 1 - 5 }$n(b)$ & 93,898 & 522,746 & 562,284 & 899,059 & $1,034,278$ & $1,179,568$ & \\
\end{tabular}

THEOREM 2. For all $n \geq 3$, we have

$$
\log g(n) \leq \sqrt{n \log n}\left(1+\frac{\log \log n-a}{2 \log n}\right)
$$

with $a=0.975$.

To prove these two theorems, we construct a subset $G$ of $g(\mathbb{N})$ analogous to the set of superior highly composite numbers introduced by Ramanujan in his study of the high values of the number of divisors of an integer (cf. [13], [11], and [12]). We also need sharp effective estimates involving primes. Finally, we use a computer to improve the values of the various constants in the lower range of $n$.

Clearly, from (1.3), we see that Theorem 2 is valid for $a=1$ and for all $n$ large enough. In fact, we think that Theorem 2 holds with $a=1$, for all $n \geq 4$. But to prove that, we need an estimation for Chebyshev's function $\theta(x)=\sum_{p \leq x} \log p$ of the form

$$
|\theta(x)-x|<c x / \log ^{2} x, \quad x>x_{0} .
$$

Schoenfeld (cf. [16]) has proved such an inequality with $c=8.072$ and $x_{0}=1$, but this value of $c$ is not small enough for our purpose.

In [10], an algorithm has been given to calculate $g(n)$ by a method of dynamical programming. Recently, this algorithm was implemented by F. Morain (cf. [8]). He has calculated exact values of $g(n)$ up to $n=32,000$ and approximate values with 16 significant digits up to $n=2,626,000$. A table up to 300 is printed in [9].

Let $P(N)$ be the greatest prime divisor of $N$. It is of some interest for the above algorithm to have a good upper bound for $P(g(n))$. In [9], it is proved that

$$
P(g(n)) \sim \log g(n) \sim \sqrt{n \log n} .
$$

Actually, the proof gives

$$
P(g(n))=\sqrt{n \log n}(1+O(1 / \log \log \log n)) .
$$


We shall prove here

THEOREM 3. We have

$$
P(g(n))=\sqrt{n \log n}\left(1+\frac{\log \log n+O(1)}{2 \log n}\right) .
$$

If $d_{n}$ is defined by

$$
P(g(n))=d_{n} \sqrt{n \log n}
$$

then for all $n \geq 2$ we have $d_{n} \leq 2.86$.

Remark. The above upper bound for $d_{n}$ is far from being best possible. In fact, but for $d_{2}=1.69 \ldots$ and $d_{3}=1.65 \ldots$, it appears that $d_{n}$ is always smaller than $d_{215}=1.26 \ldots$ The proof of Theorem 3 is simple and does not need much computation. Up to now, we were not able to improve it significantly. The proofs of (1.7) and (1.8) are constructive, but using either of them to improve upon Theorem 3 will require very extensive computation.

Notations. The letters $p, q, P$ will denote prime numbers; $p_{k}$ will be the $k$ th prime number. We define

$$
\begin{aligned}
& S_{k}=\sum_{i=1}^{k} p_{i} \text { and } S(x)=\sum_{p \leq x} p \\
& \left.\begin{array}{rl}
\theta(x) & =\sum_{p \leq x} \log p \\
\psi(x) & =\sum_{p, m ; p^{m} \leq x} \log p
\end{array}\right\} \quad \text { Chebyshev functions } \\
& \pi(x)=\sum_{p \leq x}^{1 .}
\end{aligned}
$$

2. Properties of $g(n)$. We shall enunciate below the results about $g(n)$ that will be used in the proofs of our theorems. Most of these statements are proved in [10] or in [5].

First, let us define $l(N)$ as the additive function such that $l\left(p^{\alpha}\right)=p^{\alpha}$ and $l(1)=0$. If we write the standard factorization of the integer $M$ into primes as $M=\prod_{i=1}^{k} q_{i}^{\alpha_{i}}, \alpha_{i} \geq 1$, then

$$
l(M)=\sum_{i=1}^{k} q_{i}^{\alpha_{i}}
$$

With this definition we get

$$
g(n)=\max _{l(M) \leq n} M \quad \text { which implies } n \geq l(g(n))
$$

and

$$
N \in g(\mathbb{N}) \Leftrightarrow(M>N \Rightarrow l(M)>l(N)) .
$$

Now, we say that $N \in G$ if there exists $\rho>0$ such that

$$
\forall M \in \mathbb{N}^{*}, \quad l(M)-\rho \log M \geq l(N)-\rho \log N .
$$

It is easy to see from (2.2) that $G \subset g(\mathbb{N})$ and that

$$
\text { if } N \in G \text { then } N=g(l(N)) \text {. }
$$


When (2.3) holds, $N$ and $\rho$ are said to be associated.

For each prime $p$, we define the set of real numbers

$$
E_{p}=\left\{\frac{p}{\log p}\right\} \cup\left\{\frac{p^{2}-p}{\log p}, \ldots, \frac{p^{\alpha+1}-p^{\alpha}}{\log p}, \ldots\right\} .
$$

For two different primes $p$ and $q, E_{p} \cap E_{q}=\varnothing$, and

$$
E=\bigcup_{p} E_{p}=\left\{\rho_{1}, \ldots, \rho_{n}, \ldots\right\} \quad \text { with } \rho_{i+1}>\rho_{i}
$$

and

$$
\rho_{1}=\frac{3}{\log 3}=2.73 \ldots, \quad \rho_{2}=\frac{2}{\log 2}=2.88 \ldots, \quad \rho_{3}=\frac{5}{\log 5}=3.10 \ldots, \ldots
$$

Let $\rho$ be a real number $\geq 2 / \log 2=4 / \log 4$ and $x \geq 4$ be such that $x / \log x=\rho$. We shall define

$$
N_{\rho}=\prod_{p \leq x} p^{\alpha_{p}}
$$

with $\alpha_{p}=1$ if $p / \log p \leq \rho<\left(p^{2}-p\right) / \log p$, and $\alpha_{p}=\alpha \geq 2$ if $\left(p^{\alpha}-p^{\alpha-1}\right) / \log p \leq$ $\rho<\left(p^{\alpha+1}-p^{\alpha}\right) / \log p$.

It is not difficult to show that

$$
\lim _{M \rightarrow+\infty} l(M)-\rho \log M=+\infty,
$$

and thus, $l(M)-\rho \log M$ has an absolute minimum which is attained for an element of $G$.

If $\rho=\rho_{i}$, with $i \geq 3$, this minimum is attained for exactly two numbers, namely $N_{\rho_{i-1}}$ and $N_{\rho_{i}}$. If $\rho \notin E$ and $\rho>\rho_{2}=2 / \log 2$, then there is only one integer for which this minimum is attained, namely $N_{\rho}$ defined by (2.5).

If $\rho<\rho_{1}$, there is a unique minimum at $M=1$.

If $\rho=\rho_{1}$, the minimum is attained at two points $M=1$ and $M=3$.

If $\rho_{1}<\rho<\rho_{2}$, the minimum is unique and at $M=3$.

If $\rho=\rho_{2}$, the minimum is attained at three points, namely $M=3, M=6$, $M=12$.

From all this, we conclude that $G=\{1,3,6,12\} \cup\left\{N_{\rho_{i}}, i \geq 3\right\}$. If $G$ is ordered increasingly, and if $N$ and $N^{\prime}$ are two consecutive terms, then there exists a unique $\rho \in E$ associated both with $N$ and $N^{\prime}$. Moreover, if $\rho \in E_{p}$ then $N^{\prime}=p N$.

The definition of $\alpha_{p}$ in (2.5) implies that for $p \leq x, \alpha_{p} \geq 1$, and $p^{\alpha_{p}} \leq x$; thus we have

$$
\theta(x) \leq \log N_{\rho} \leq \psi(x)
$$

and

$$
\sum_{p \leq x} p \leq l\left(N_{\rho}\right)
$$

\section{Some Lemmas.}

LEMMA 1. let $t_{0}$ and $k$ be positive real numbers. Then

$$
\max _{t \geq t_{0}} e^{-t}\left(t-t_{0}\right)^{k}=k^{k} e^{-t_{0}-k} .
$$

The proof is an easy exercise in calculus. 
LEMMA 2. let $\phi=\phi_{\lambda, a}$ be the function defined by

$$
\phi(x)=\sqrt{x \log x}\left(1+\lambda \frac{\log \log x-a}{2 \log x}\right) .
$$

If $\lambda \geq 0$ and $a \geq 0$, then $\phi$ is increasing and concave for $x>1$.

Proof. Using the computer algebra system MACSYMA, we find

$$
\begin{aligned}
& \phi^{\prime}(x)=\frac{\lambda(\log x-1) \log \log x+2 \log ^{2} x+(2-\lambda a) \log x+\lambda(a+2)}{4 \sqrt{x}(\log x)^{3 / 2}} \\
& \phi^{\prime \prime}(x)=-\frac{\lambda\left(\log ^{2} x-3\right) \log \log x+2 \log ^{3} x+\lambda a \log ^{2} x+2 \log x+\lambda(3 a+8)}{8 x^{3 / 2}(\log x)^{5 / 2}} .
\end{aligned}
$$

Let us set $y=\log x$ and

$$
Y=\lambda\left(y^{2}-3\right) \log y+2 y^{3}+\lambda a y^{2}+2 y+\lambda(3 a+8) .
$$

For $0<y<1$ and $y>\sqrt{3}$, the first term in $Y$ is positive, and thus $Y$ is positive. For $1 \leq y \leq \sqrt{3}$ we have

$$
Y \geq 2+\lambda a+2+\lambda(3 a+8)+\lambda(1-3) \log \sqrt{3} \geq \lambda(8-\log 3)>0,
$$

and so $\phi^{\prime \prime}(x)$ is clearly negative. Then $\phi^{\prime}$ is decreasing, and as $\lim _{x \rightarrow+\infty} \phi^{\prime}(x)=0$, $\phi^{\prime}(x)$ is positive for all $x>1$.

LEMMA 3. If $S(x)=\sum_{p \leq x} p$, we have

$$
\begin{array}{ll}
\text { (i) } S(x) \geq \frac{x^{2}}{2 \log x}\left(1+\frac{1}{2 \log x}\right) & \text { for } 302,971 \leq x \leq \exp (90), \\
\text { (ii) } S(x) \geq \frac{x^{2}}{2 \log x}\left(1+\frac{0.477}{\log x}\right) & \text { for } x \geq 70,001 \\
\text { (iii) } S(x) \geq \frac{x^{2}}{2 \log x} \exp \left(\frac{0.475}{\log x}\right) & \text { for } x \geq p_{300,000}=4,256,233 .
\end{array}
$$

Proof. First, we have from [5]

$$
\begin{aligned}
S(x) & =\operatorname{li}\left(x^{2}\right)+O\left(x^{2} e^{-a \sqrt{\log x}}\right) \quad(\text { with } a>0) \\
& =\frac{x^{2}}{2 \log x}+\left(1+\frac{1}{2 \log x}+O\left(\frac{1}{\log ^{2} x}\right)\right) .
\end{aligned}
$$

The proof of (i) and (ii) is in [6] and is an improvement of Lemma 6 of [3]. It was obtained by considering the Stieltjes integral

$$
\sum_{p \leq x} p=2+\int_{e}^{x} \frac{t}{\log t} d \theta(t)
$$

and using effective estimations of $\theta$ due to J. B. Rosser and L. Schoenfeld (cf. [15]). Further, by Taylor's formula, we have $\exp (u) \leq 1+u+u^{2} \exp \left(u_{0}\right) / 2$ for $0 \leq u \leq u_{0}$. If we set $u=0.475 / \log x$, then we have $u \leq u_{0}=0.032$ for $x \geq p_{300,000}$ and $u \leq u_{0}=0.0072$ for $x \geq \exp (66)$. Since $(0.475)^{2} \exp \left(u_{0}\right) / 2 \log x$ is less than 0.008 for $x \geq p_{300,000}$, and less than 0.0018 for $x \geq 10^{11}$, the proof of (iii) is complete. 
LEMMA 4. Let $p_{k}$ be the kth prime and $S_{k}=\sum_{i \leq k} p_{i}$. For $k \geq 3,688$ we have
(i) $S_{k} \leq \frac{k^{2}}{2}\left(\log k+\log \log k-3 / 2+\frac{1.866 \log \log k}{\log k}\right)$,
(ii) $S_{k} \leq \frac{k^{2}}{2}(\log k+\log \log k-1.4416)$.

Proof. Cf. [6], where an improvement of the estimation of $p_{k}$ given in [14] is applied.

We may compare Lemma 4 with the asymptotic expansion

$$
S_{k}=\frac{k^{2}}{2}(\log k+\log \log k-3 / 2+\log \log k / \log k+O(1 / \log k)) .
$$

LEMMA 5. With the notation of Section 2, let $N^{\prime}$ belong to $G$ and be associated with $\rho^{\prime}$. We assume that $N>N^{\prime}$ and that there exists $\rho>0$ such that for all $M \geq N^{\prime}$ we have

$$
l(M)-\rho \log M \geq l(N)-\rho \log N
$$

Then we have

(i) $\rho \geq \rho^{\prime}$

(ii) $N \in G$ and $N$ and $\rho$ are associated.

Proof. We have from (2.3)

$$
l(N)-\rho^{\prime} \log N \geq l\left(N^{\prime}\right)-\rho^{\prime} \log N^{\prime} .
$$

Now, (3.1) with $M=N^{\prime}$ gives

$$
l\left(N^{\prime}\right)-\rho \log N^{\prime} \geq l(N)-\rho \log N
$$

and thus

$$
\rho^{\prime} \log \left(N / N^{\prime}\right) \leq l(N)-l\left(N^{\prime}\right) \leq \rho \log \left(N / N^{\prime}\right)
$$

which proves (i). To prove (ii), it suffices to show (3.1) for $M<N^{\prime}$. Suppose that $M<N^{\prime}$. Then we shall have

$$
\begin{aligned}
l(M)-\rho \log M & =l(M)-\rho^{\prime} \log M-\left(\rho-\rho^{\prime}\right) \log M \\
& \geq l\left(N^{\prime}\right)-\rho^{\prime} \log N^{\prime}-\left(\rho-\rho^{\prime}\right) \log M \\
& =l\left(N^{\prime}\right)-\rho \log N^{\prime}+\left(\rho-\rho^{\prime}\right) \log \left(N^{\prime} / M\right) \\
& \geq l(N)-\rho \log N+\left(\rho-\rho^{\prime}\right) \log \left(N^{\prime} / M\right),
\end{aligned}
$$

where we have used (2.3) and (3.2). But in the above chain of inequalities, the last term in the right-hand side is nonnegative.

This lemma is similar to Lemma 4 of [11].

LEMMA 6. (i) For all $n$ such that $1,179,568 \leq n \leq S_{4,399}=87,221,664$ we have, with $\phi$ defined in Lemma 2 ,

$$
\log g(n) \geq \phi_{1, b}(n) \quad \text { with } b=1.16 \text {. }
$$

(ii) For all $n$ such that $4 \leq n \leq n_{1}$ with $n_{1}=614,219,626,329$ we have

$$
\log g(n)<\phi_{1,1}(n) .
$$


Proof. (i) For all pairs of consecutive elements of $G, N<N^{\prime}$, we define $a^{\prime}=$ $a^{\prime}\left(N, N^{\prime}\right)$ by

$$
\log N=\phi_{1, a^{\prime}}\left(l\left(N^{\prime}\right)\right) .
$$

Now suppose that $l(N) \leq n \leq l\left(N^{\prime}\right)$. Since $g$ is nondecreasing, by (2.4) we shall have

$$
N \leq g(n) \leq N^{\prime}
$$

By Lemma 2, $\phi_{1, a^{\prime}}$ is increasing and

$$
\log g(n) \geq \log N=\phi_{1, a^{\prime}}\left(l\left(N^{\prime}\right)\right) \geq \phi_{1, a^{\prime}}(n) .
$$

The elements of $G$ are easy to calculate. With a computer, we check that $a^{\prime} \leq 1.16$ for all pairs $\left(N, N^{\prime}\right)$ such that $3,705,101 \leq l(N) \leq n_{1}$, and this proves (i) for all $n$ in the range $3,705,101 \leq n \leq n_{1}$.

For smaller $n$, we improve the above trick by introducing $N^{*}=N Q$, where $Q$ is the smallest prime dividing $N$ with exponent 1 . When $N^{*}$ verifies $N^{*}<N^{\prime}$ we define $a^{\prime \prime}$ and $a^{\prime \prime \prime}$ by

$$
\log N=\phi_{1, a^{\prime \prime}}\left(l\left(N^{*}\right)\right) \text { and } \log N^{*}=\phi_{1, a^{\prime \prime \prime}}(l(N)) .
$$

It is then not difficult to show that

$$
\begin{array}{ll}
\log g(n) \geq \phi_{1, a^{\prime \prime}}(n) & \text { for } l(N) \leq n \leq l\left(N^{*}\right), \\
\log g(n) \geq \phi_{1, a^{\prime \prime \prime}}(n) & \text { for } l\left(N^{*}\right) \leq n \leq l\left(N^{\prime}\right) .
\end{array}
$$

Calculating $a^{\prime \prime}$ and $a^{\prime \prime \prime}$ proves (i) for 2, 294, $185 \leq n \leq 3,705,101$.

To conclude the proof of (i), F. Morain (cf. [8]) has calculated an approximate value of $g(n)$ for $1 \leq n \leq 2,626,000$, with the algorithm of [11]. This computation has shown (i) for $1,179,568 \leq n \leq 2,626,000$, and moreover has given the various pairs $\left(b, n_{b}\right)$ tabulated in Theorem 1 .

(ii) We shall write $\phi=\phi_{1,1}$. From Lemma 2, $\phi$ has an inverse function $\phi^{-1}$ which is increasing and convex. First we check with a computer that for all $N \in G$ such that $7 \leq l(N) \leq n_{1}$ we have

$$
\log N<\phi(l(N))
$$

Now suppose that $N$ and $N^{\prime}$ are two consecutive elements of $G, \rho$ their common associated parameter, verifying (3.3), and

$$
\log N^{\prime}<\phi\left(l\left(N^{\prime}\right)\right) .
$$

Furthermore, let $l(N) \leq n<l\left(N^{\prime}\right)$. As above, we shall have $N \leq g(n)<N^{\prime}$. From the convexity of $\phi^{-1}(x)-\rho x$ we deduce that one of the two following relations holds:

$$
\begin{gathered}
\phi^{-1}(\log g(n))-\rho \log g(n) \leq \phi^{-1}(\log N)-\rho \log N \\
\phi^{-1}(\log g(n))-\rho \log g(n) \leq \phi^{-1}\left(\log N^{\prime}\right)-\rho \log N^{\prime} .
\end{gathered}
$$

Suppose that (3.5) holds. With (3.3) we obtain

$$
\phi^{-1}(\log g(n))-\rho \log g(n)<l(N)-\rho \log N \leq l(g(n))-\rho \log g(n),
$$

since $N \in G$ and is associated with $\rho$. By $(2.1)$ this implies $\phi^{-1}(\log g(n))<n$, which is equivalent to $\log g(n)<\phi(n)$. It remains to check (ii) for $n=4,5,6$.

Remark. The value of $n_{1}$ is in fact $l\left(N_{\rho}\right)$ for $\rho=278,843.2$. The largest prime factor of $N_{\rho}$ is $p_{300000}=4,256,233$. 
4. The Lower Bound.

PROPOSITION 1. For $k \geq 4,451$, that is, $p_{k} \geq 42,569$, we have

(i) $\theta^{2}\left(p_{k-1}\right) \geq S_{k} \log S_{k}\left(1+\left(\log \log S_{k}-b^{\prime}\right) / \log S_{k}\right)$ with $b^{\prime}=1.1168$.

For $k \geq 4,399$, that is, $p_{k} \geq 42,071$, we have

(ii) $\theta\left(p_{k-1}\right) \geq \phi_{1, b}\left(S_{k}\right)$ with $b=1.16$.

Proof. The cases $k \leq 600,000$ are verified by computation. We therefore assume that $k \geq 600,000$. Let $\alpha_{1}=1.4416$, the constant occurring in Lemma 4 . We define $b^{\prime}=2\left(2-\alpha_{1}\right)=1.1168$ and for simplicity write $L=\log k$ and $L_{2}=\log \log k$.

From Lemma 4 we have

$$
\begin{aligned}
S_{k} & \leq\left(k^{2} / 2\right)\left(L+L_{2}-\alpha_{1}\right), \\
\log S_{k} & \leq 2 L-\log 2+L_{2}+\log \left(1+\left(L_{2}-\alpha_{1}\right) / L\right) \\
& \leq 2 L+L_{2}-\log 2+\left(L_{2}-\alpha_{1}\right) / L \\
& =2 L\left(1+L_{2} / 2 L-\log 2 / 2 L+\left(L_{2}-\alpha_{1}\right) / 2 L^{2}\right), \\
\log \log S_{k} & \leq L_{2}+\log 2+\left(L_{2}-\log 2\right) / 2 L+\left(L_{2}-\alpha_{1}\right) / 2 L^{2},
\end{aligned}
$$

and then

$\log S_{k}+\log \log S_{k} \leq 2 L+2 L_{2}+\left(3 L_{2}-2 \alpha_{1}-\log 2\right) / 2 L+\left(L_{2}-\alpha_{1}\right) / 2 L^{2}$.

For $k \geq 600,000$, we have $\left(L_{2}-\alpha_{1}\right) L \leq 0.087$, which implies

$$
\log S_{k}+\log \log S_{k} \leq 2 L+2 L_{2}+\left(3 L_{2}-\beta\right) / 2 L
$$

with $\beta=3.489<2 \alpha_{1}+\log 2-0.087$.

The right-hand side of (i), which is equal to $S_{k}\left(\log S_{k}+\log \log S_{k}-b^{\prime}\right)$, is thus smaller than

$$
k^{2}\left(L+L_{2}-\alpha_{1}\right)\left(L+L_{2}-b^{\prime} / 2+\left(3 L_{2}-\beta\right) / 4 L\right) .
$$

We shall now use the following inequality (cf. [14, Theorem 7]):

$$
\theta\left(p_{k}\right) \geq k\left(L+L_{2}-1+\left(L_{2}-\gamma\right) / L\right)
$$

with $\gamma=2.1454$. Further, we deduce from $p_{k} \leq k\left(L+L_{2}\right)$ (cf. $[15$, p. 69]) that

$$
\left(\log p_{k}\right) / k \leq\left(L+L_{2}+L_{2} / L\right) / k \leq 3.6 \cdot 10^{-4} / L .
$$

Then, with (4.2) we obtain

$$
\theta\left(p_{k-1}\right)=\theta\left(p_{k}\right)-\log p_{k} \geq k\left(L+L_{2}-1+\left(L_{2}-\gamma^{\prime}\right) / L\right),
$$

with $\gamma^{\prime}=2.1458$, and

$$
\theta^{2}\left(p_{k-1}\right) \geq k^{2}\left(\left(L+L_{2}\right)^{2}-2\left(L+L_{2}\right)+1+2\left(L_{2}-\gamma^{\prime}\right)\left(1+\left(L_{2}-1\right) / L\right)\right) .
$$

So, to prove (i), it is enough to prove that

$$
\alpha_{1} b^{\prime} / 2+\left(3 L_{2}-\beta\right)\left(1+\left(L_{2}-\alpha_{1}\right) / L\right) / 4 \leq 1+2\left(L_{2}-\gamma^{\prime}\right)\left(1+\left(L_{2}-1\right) / L\right),
$$

because $b^{\prime}=2\left(2-\alpha_{1}\right)$. This inequality is equivalent to

$$
w_{0} L L_{2}-w_{1} L+w_{2} L_{2}^{2}-w_{3} L_{2}+w_{4} \geq 0
$$


with

$$
\begin{aligned}
& w_{0}=w_{2}=5 / 4, \\
& w_{1}=2 \gamma^{\prime}+\alpha_{1} b^{\prime} / 2-\beta / 4-1=3.22433944, \\
& w_{3}=2 \gamma^{\prime}-3 \alpha_{1} / 4+2-\beta / 4=4.33815, \\
& w_{4}=2 \gamma^{\prime}-\alpha_{1} \beta / 4=3.0341644 .
\end{aligned}
$$

Setting $t=L_{2}, L=e^{t}$, the study of the function $W(t)=w_{0} t e^{t}-w_{1} e^{t}+w_{2} t^{2}-$ $w_{3} t+w_{4}$ is easy. As $3 w_{0}>w_{1}, W^{\prime \prime}(t)>0$ for $t \geq 1$. So $W^{\prime}(t)$ is increasing and is positive for $t \geq 2$. Then we have

$$
W(t) \geq W(\log \log 600,000) \geq W(2.588) \geq 0.32 .
$$

So (i) is proved.

For (ii) we write

$$
1+\frac{\log \log S_{k}-b^{\prime}}{\log S_{k}}=\left(1+\frac{\log \log S_{k}-b}{2 \log S_{k}}\right)^{2}+\frac{b-b^{\prime}}{\log S_{k}}-\frac{\left(\log \log S_{k}-b\right)^{2}}{4 \log ^{2} S_{k}}
$$

However, by Lemma 1 , the last term is in absolute value smaller than $e^{-b-2} / \log S_{k}$. The values of $b$ and $b^{\prime}$ have been chosen such that $b>b^{\prime}+e^{-b-2}$, thus (ii) follows from (i) for $k \geq 4,451$.

Proof of Theorem 1. We define $k=k(n)$ such that $S_{k-1} \leq n<S_{k}$, and we consider a permutation of $\sigma_{n}$ whose lengths of the cycles are $2,3, \ldots, p_{k-1}$ and 1 , $n-S_{k-1}$ times. The order of such a permutation is $\prod_{i=1}^{k-1} p_{i}=\exp \left(\theta\left(p_{k-1}\right)\right)$, and it follows from (ii) of Proposition 1 that

$$
\log g(n) \geq \theta\left(p_{k-1}\right) \geq \phi_{1, b}\left(S_{k}\right)
$$

for $n \geq S_{4,399}=87,221,664$. For smaller $n$ 's we apply Lemma 6 .

5. The Upper Bound. In [4], the method used to prove (1.5) was the following: First (1.5) is proved when $g(n) \in G$. Then the concavity of $\phi$ (cf. Lemma 2) is used to show (1.5) for all $n$. When $g(n) \in G$, the proof of (1.5) is based on the pair of inequalities (cf. (2.6) and (2.7))

$$
\log g(n) \leq \psi(x) \quad \text { and } \quad n \geq \sum_{p \leq x} p
$$

A lower bound for the above sum is given by Lemma 3 .

We shall use here a somewhat more complicated idea, close to the proof of (1.4) in [3]. This idea gives a better effective result, mainly because we have to use Lemma 3 to estimate $\log \left(\sum_{p \leq x} p\right)$ rather than $\sum_{p \leq x} p$.

From now on, $a$ will be fixed and equal to 0.975 . For all $n \geq 2$, we define $\lambda_{n}$ by

$$
\log g(n)=\phi_{\lambda_{n}, a}(n) \text {. }
$$

It follows from Lemma 6 for $n_{0} \leq n \leq n_{1}$, with $n_{0}=4$ and $n_{1}=614,219,626,329$, that we have $\lambda_{n} \leq 1$. On the other hand, (1.3) implies $\lim _{n \rightarrow+\infty} \lambda_{n}=1$. We want to prove that $\lambda_{n} \leq 1$ for all $n \geq n_{1}$.

We shall suppose that there exists $n \geq n_{1}$ with $\lambda_{n}>1$ and show that this is impossible. Indeed, in that case, let $\lambda=\max _{n \geq n_{1}} \lambda_{n}>1$. There will be an $n$ such that

$$
\log g(n)=\phi(n)
$$


and for $m \geq n_{0}$

$$
\log g(m) \leq \phi(m), \quad \text { where we write } \phi \text { for } \phi_{\lambda, a} .
$$

We shall show that (5.1) and (5.2) imply that $g(n)$ belongs to $G$. For this, we use Lemma 5 with $\rho^{\prime}=4, N^{\prime}=420 \in G, N=g(n)$ and $\rho=1 / \phi^{\prime}(n)$. In order to check (3.1), we write, for $M \geq N^{\prime}$,

$$
l(M)-\rho \log M=\left[l(M)-\phi^{-1}(\log M)\right]+\left[\phi^{-1}(\log M)-\rho \log M\right] .
$$

As $l\left(N^{\prime}\right)=19$ and $M \geq N^{\prime}$, we have from (2.2) that $m=l(M) \geq 19$, and from (2.1) and (5.2) we get

$$
\log M \leq \log g(m) \leq \phi(m)=\phi(l(M)) .
$$

Thus, the first bracket in (5.3) is positive.

Further, by Lemma 2, the function $v \rightarrow v-\rho \phi(v)$ is convex, and with our choice of $\rho$, has an absolute minimum for $v=n$. So, for all $v>1$, we have

$$
v-\rho \phi(v) \geq n-\rho \phi(n),
$$

and then, setting $v=\phi^{-1}(\log M)$, and using (5.1), the second bracket of (5.3) is greater than $n-\rho \log g(n) \geq l(N)-\rho \log N$. Thus, condition (3.1) is verified, and from $\lambda>1, g(n)=N$ belongs to $G$ and is associated with $\rho=1 / \phi^{\prime}(n)$. The conclusion of the proof of Theorem 2 now follows from

PROPOSITION 2. For $a=0.975$ and $\lambda \geq 1$ there is no $n, n \geq n_{1}$, for which $\log g(n)=\phi_{\lambda, a}(n)$ and $g(n) \in G$ is associated with $1 / \phi_{\lambda, a}^{\prime}(n)$.

Proof. We set $\phi=\phi_{\lambda, a}$ and $\rho=1 / \phi_{\lambda, a}^{\prime}(n)$. Let $n$ be such that $\log g(n)=\phi(n)$. First we define $x$ by $\rho=x / \log x$. As $g(n)$ belongs to $G$, is associated with $\rho$, and $n \geq n_{1}$, this implies $x \geq p_{300,000}=4,256,233$. Then by (2.6), we obtain

$$
\phi(n)=\log g(n) \leq \psi(x) \leq x(1+c / \log x)
$$

with $c=0.0092$ for $p_{300,000}<x<1.04 \cdot 10^{7}$ and $c=0.00777$ for $x \geq 1.04 \cdot 10^{7}$. The first value is obtained from $\theta(x)<x$ and the inequality $\psi(x)<\theta(x)+x^{1 / 2}+3 x^{1 / 3}$ for $x \leq 10^{16}$ [15, p. 73], the second follows from [16, p. 357].

We deduce from (5.4) that

$$
\phi(n) \phi^{\prime}(n)=\phi(n) / \rho \leq \log x+c .
$$

Now we shall get a lower bound for $\phi_{n} \phi_{n}^{\prime}$. First we observe (cf. Lemma 2 above) that the coefficient of $\lambda$ in the numerator of $\phi^{\prime}(n)$ is

$(\log n-1) \log \log n-a \log n+a+2 \geq \log n(\log \log n-a-1) \geq 0 \quad$ for $n \geq n_{1}$.

So, we shall have

$$
\phi(n) \phi^{\prime}(n)=\phi_{\lambda, a}(n) \phi_{\lambda, a}^{\prime}(n) \geq \phi_{1, a}(n) \phi_{1, a}^{\prime}(n) .
$$

Setting $y=\log n$, we have $\phi_{1, a}(n) \phi_{1, a}^{\prime}(n)=E(y)$ with

$$
E(y)=\frac{(y-1) \log y+2 y^{2}+(2-a) y+a+2}{4 y}\left(1+\frac{\log y-a}{2 y}\right) .
$$


Further,

$$
E(y) \geq \frac{y}{2}+\frac{\log y}{4}+\frac{2-a}{4}-\frac{\log y}{4 y}+\frac{a+2}{4 y}+\frac{\log y-a}{2 y}\left(\frac{y}{2}+\frac{\log y}{4}+\frac{2-a-\eta}{4}\right)
$$

with $\eta=\max \{(\log y-a+2) / y\}$ which, by Lemma 1 , is $\leq e^{-a-3} \leq 1-a$. Then,

$$
2 E(y) \geq y+\log y+1-a+\frac{Q(\log y)}{4 y}
$$

where

$$
Q(Y)=Y^{2}-(a+1) Y+a+4 .
$$

By (2.1) and (2.7), $n \geq \sum_{p \leq x} p$, and by Lemma 3 ,

$$
y>2 \log x-L_{2}-\log 2+0.475 / u
$$

where $L_{2}$ stands for $\log \log x$. We now set for $u \geq 15$,

$$
f(u)=2 u-\log u-\log 2+0.475 / u
$$

and we shall show that, if $z=f(u)$, and $u \geq 15$, then $u \leq v$, where $v$ is defined by

$$
v=z / 2+(\log z) / 2+(\log z) / 2 z-0.415 / z .
$$

As $f$ is increasing, it suffices to prove that $f(v) \geq z$. We observe that

$$
z \geq f(15) \geq 26.63, \quad \exp (\log z / z) \geq 1+\log z / z+\log z / z^{2},
$$

since $\log z>2$, and from this inequality we deduce that

$$
\log v \leq \log (z / 2)+\log z / z
$$

Furthermore, we have

$$
\frac{1}{1+\log z / z+\log z / z^{2}-0.83 / z^{2}} \geq 1-\frac{\log z}{z},
$$

and from (5.10), (5.11) and (5.12) we see that

$$
\begin{aligned}
f(v) & \geq z+\log z+\frac{\log z}{z}-\frac{0.83}{z}-\log \frac{z}{2}-\frac{\log z}{z}-\log 2+\frac{0.95}{x}\left(1-\frac{\log z}{z}\right) \\
& =z+\frac{1}{z}\left(0.12-0.95 \frac{\log z}{z}\right) \geq z .
\end{aligned}
$$

Now, from (5.9) and (5.10), we deduce that

$$
\log x \leq \frac{y}{2}+\frac{\log y}{2}+\frac{\log y}{2 y}-\frac{0.415}{y} \text { for } \log x \geq 15 .
$$

Then (5.7) gives

$$
\begin{aligned}
E(y)-\log x & \geq \frac{1-a}{2}+\frac{Q(\log y)-4 \log y+3.32}{8 y} \\
& =0.0125+\frac{1}{8 y}\left((\log y-2.9875)^{2}-0.63015625\right) \\
& \geq 0.0125-\frac{0.63015625}{8 y} .
\end{aligned}
$$

We observe that $x \geq 4,256,233=p_{300,000}$, which implies $\log x \geq 15, y \geq f(\log x) \geq$ 26.63 and then

$$
E(y)-\log x \geq 0.00954
$$

which contradicts (5.5). 


\section{Proof of Theorem 3.}

LEMMA 7. Let $N$ and $N^{\prime}$ be two consecutive elements of $G$, with $N \geq 12$, and $\rho=x / \log x$ their common parameter. Define, for any $M$, the benefit of $M$ by

$$
\text { ben } M=l(M)-l(N)-\rho \log (M / N) .
$$

Then we have for $l(N) \leq n \leq l\left(N^{\prime}\right)$

(i) ben $g(n) \leq x$,

(ii) ben $g(n)=O(x / \log x)$.

Proof. As $N \in G$, by (2.4), $N=g(l(N)) \leq g(n)$ because $g$ is nondecreasing and by $(2.1)$,

$$
\text { ben } g(n) \leq l(g(n))-l(N) \leq n-l(N) \leq l\left(N^{\prime}\right)-l(N)
$$

Now $N^{\prime}=p N$, and if $p$ does not divide $N$, then $\rho=p / \log p$ and $x=p=$ $l\left(N^{\prime}\right)-l(N)$. If $p^{\alpha} \| N$ then

$$
l\left(N^{\prime}\right)-l(N)=p^{\alpha+1}-p^{\alpha}=\rho \log p \leq \rho \log x=x
$$

and (i) is proved; (ii) is Lemma D of [5].

Unfortunately, it does not seem easy to replace the $O$ in (ii) by a reasonably small constant.

LEMMA 8. With the same notation as in Lemma 7, if a prime $P$ divides $g(n)$, then

(i) $P \leq 2 x-x /(\log x-1)$,

(ii) $P \leq x+O(x / \log x)$.

Proof. Let $N$ be defined by $(2.5)$ and $g(n)=\prod_{p} p^{\beta_{p}}$. We shall have

$$
\operatorname{ben} g(n)=\sum_{p}\left(l\left(p^{\beta_{p}}\right)-l\left(p^{\alpha_{p}}\right)-\rho\left(\beta_{p}-\alpha_{p}\right) \log p\right) \text {. }
$$

From the definition of $\alpha_{p}$ in (2.5), each term in the above sum is nonnegative.

Further, if $P$ divides $N$, then $P \leq x$ and Lemma 8 is proved. If $P$ does not divide $N$ and $P>x$, then $\alpha_{p}=0$, and from (6.1) and Lemma 7(i) we deduce

$$
x \geq P^{\beta_{P}}-\beta_{P} \rho \log P \geq \beta_{P}(P-\rho \log P) \geq P-\rho \log P .
$$

By the mean value theorem,

$$
\begin{aligned}
P-\rho \log P & =\log P\left(\frac{P}{\log P}-\frac{x}{\log x}\right) \\
& \geq \log P(P-x) \frac{\log P-1}{\log ^{2} P} \geq(P-x)(1-1 / \log x)
\end{aligned}
$$

and thus $P \leq x+x /(1-1 / \log x)$.

If, in the above proof, we use (ii) of Lemma 7 instead of (i), we obtain (ii).

Proof of Theorem 3. To prove (1.8), observe first that, with the notation of Lemma 7, we have

$$
P(g(n))=x+O(x / \log x)
$$


Indeed, from Lemma 8(ii), the right-hand side of (6.2) is an upper bound for $P(g(n))$. It can be proved similarly that it is also a lower bound. Secondly, $N \leq g(n) \leq N^{\prime}$, and by $(2.6)$,

$$
\theta\left(x^{-}\right) \leq \log N \leq \log g(n) \leq \log N^{\prime} \leq \psi(x),
$$

where $\theta\left(x^{-}\right)=\lim _{t \rightarrow x, t<x} \theta(t)$. (6.3) implies

$$
\log g(n)=x+O(x / \log x) .
$$

Then (6.2) and (1.3) give the result.

To prove (1.9), set

$$
\begin{gathered}
x_{0}=101, \quad \rho_{0}=x_{0} / \log x_{0}=21.88 \ldots \\
N_{\rho_{0}}=2^{4} 3^{3} 5^{2} 7^{2} \prod_{11 \leq p \leq 101} p \text { and } l\left(N_{\rho_{0}}\right)=n_{0}=1,261 .
\end{gathered}
$$

For $n \geq n_{0}$, we define $N$ and $N^{\prime}$ as the two consecutive elements of $G$ such that $l(N) \leq n<l\left(N^{\prime}\right)$, and the $x$ mentioned in Lemma 7 will be $\geq x_{0}$. Then by Lemma 8,

$$
P(g(n)) \leq x\left(2+1 /\left(\log x_{0}-1\right)\right) \leq 2.28 x .
$$

Further, for $x \geq x_{0}$ we have $\theta\left(x^{-}\right) \geq 0.84 x$ (cf. [15, p. 71]). By $(6.3), \log g(n) \geq$ $\theta\left(x^{-}\right)$, and with (1.4),

$$
P(g(n)) \leq \frac{2.28 \times 1.05314}{0.84} \sqrt{n \log n} \leq 2.86 \sqrt{n \log n} .
$$

Thus, (1.9) is proved for $n \geq n_{0}=1,261$. For smaller $n$ 's, we compute $g(n)$ with the algorithm described in [11], using all primes up to 1,261. The table below gives for each $p$ the smallest $n$, say $n_{p}$, such that $p$ divides $g(n)$, and the quotient $p / \sqrt{n_{p} \log n_{p}}$. We observe that $n_{p}$ is not always increasing.

\begin{tabular}{|c|c|c|c|c|c|c|c|r|}
\hline$p$ & 2 & 3 & 5 & 7 & 11 & 13 & 17 & 19 \\
\hline$n_{p}$ & 2 & 3 & 8 & 14 & 27 & 32 & 57 & 62 \\
\hline$p / \sqrt{n_{p} \log n_{p}}$ & 1.699 & 1.653 & 1.226 & 1.152 & 1.167 & 1.235 & 1.120 & 1.188 \\
\hline
\end{tabular}

\begin{tabular}{|c|c|c|c|c|c|c|c|c|}
\hline$p$ & 23 & 29 & 31 & 37 & 41 & 43 & 47 & 53 \\
\hline$n_{p}$ & 93 & 118 & 128 & 178 & 213 & 215 & 297 & 346 \\
\hline$p / \sqrt{n_{p} \log n_{p}}$ & 1.121 & 1.223 & 1.244 & 1.219 & 1.214 & 1.266 & 1.143 & 1.179 \\
\hline
\end{tabular}

\begin{tabular}{|c|c|c|c|c|c|c|c|c|}
\hline$p$ & 59 & 61 & 67 & 71 & 73 & 79 & 83 & 89 \\
\hline$n_{p}$ & 399 & 429 & 519 & 510 & 586 & 687 & 780 & 920 \\
\hline$p / \sqrt{n_{p} \log n_{p}}$ & 1.207 & 1.197 & 1.177 & 1.260 & 1.195 & 1.180 & 1.152 & 1.124 \\
\hline
\end{tabular}




\begin{tabular}{|c|c|c|c|c|c|c|c|c|}
\hline$p$ & 97 & 101 & 103 & 107 & 109 & 113 & 127 & 131 \\
\hline$n_{p}$ & 946 & 1,033 & 1,106 & 1,128 & 1,209 & 1,192 & 1,614 & 1,618 \\
\hline$p / \sqrt{n_{p} \log n_{p}}$ & 1.205 & 1.193 & 1.170 & 1.202 & 1.177 & 1.230 & 1.164 & 1.198 \\
\hline
\end{tabular}

Département de Mathématiques Université de Limoges 123, rue Albert Thomas 87060 Limoges, France E-mail: Massias@FRGREN81

Département de Mathématiques Universite Claude Bernard (Lyon I) 69622 Villeurbanne Cedex, France E-mail: JLNicola@FRGREN81

Département de Mathématiques Université de Limoges 123, rue Albert Thomas 87060 Limoges, France E-mail: GRobin@FRGREN81

1. E. LANDAU, "Über die Maximalordnung der Permutationen gegebenen Grades," Arch. Math. Phys. Ser. 3, v. 5, 1903, pp. 92-103.

2. E. LANDAU, Handbuch der Lehre von der Verteilung der Primzahlen, Bände 1 und 2, Chelsea, Leipzig und Berlin, 1909 (2te Auflage, New York, 1953).

3. J. P. MASSIAS, "Majoration explicite de l'ordre maximum d'un élément du groupe symétrique," Ann. Fac. Sci. Toulouse Math. v. 6, 1984, pp. 269-280.

4. J. P. Massias, Ordre Maximum d'un Élément du Groupe Symétrique et Applications, Thèse de $3^{\text {ème }}$ cycle, Université de Limoges, 1984.

5. J. P. MASSIAS, J. L. NiCOLAS \& G. ROBIN, "Evaluation asymptotique de l'ordre maximum d'un élément du groupe symétrique," Acta Arith., v. 50, 1988, pp. 221-242.

6. J. P. MASSIAS \& G. ROBIN, "Calculs effectifs sur le $k^{\text {eme }}$ nombre premier." (To appear.)

7. W. MILLER, "The maximum order of an element of a finite symmetric group," Amer. Math. Monthly, v. 94, 1987, pp. 497-506.

8. F. Morain, Tables sur la Fonction g(n), Département de Math., Université de Limoges, 1988.

9. J. L. NiCOlas, "Ordre maximal d'un élément du groupe des permutations et "highly composite numbers"," Bull. Soc. Math. France, v. 97, 1969, pp. 129-191.

10. J. L. Nicolas, Calcul de l'Ordre Maximum d'un Élément du Groupe Symétrique $S_{n}$, R.A.I.R.O. $3^{\mathrm{e}}$ année, $\mathrm{n}^{\circ} \mathrm{R}-2,1969$, pp. 43-50.

11. J. L. Nicolas \& G. ROBIN, "Majorations explicites pour le nombre de diviseurs de $n$," Canad. Math. Bull., v. 26(4), 1983, pp. 485-492.

12. J. L. NiCOLAS, Highly Composite Numbers, Proceedings of Ramanujan Conference, Urbana, June 1987, Academic Press. (To appear.)

13. S. Ramanujan, "Highly composite numbers," Proc. London Math. Soc. Ser. 2, v. 14, 1915, pp. 347-400; Collected Papers, Chelsea, 1927, pp. 78-128.

14. G. RoBIN, "Estimation de la fonction de Tchebycheff $\theta$ sur le $k$-ième nombre premier et grandes valeurs de la fonction $\omega(n)$, nombre de diviseurs premiers de $n$," Acta Arith., v. 42, 1982, pp. 367-389.

15. J. B. Rosser \& L. SCHOENFELD, "Approximate formulas for some functions of prime numbers," Illinois J. Math., v. 6, 1962, pp. 64-94

16. L. SCHOENFELD, "Sharper bounds for the Chebyshev functions $\theta(x)$ and $\psi(x)$. II," Math. Comp., v. 30, 1976, pp. 337-360. 